\title{
Erratum
}

\section{What Does Halobacterium Tell us About Photoreception?}

\section{E. Hildebrand}

Institut für Neurobiologie, Kernforschungsanlage Jülich,

Postfach 1913, D-5170 Jülich, Federal Republic of Germany

Biophys. Struct. Mechanism 3, 69-77 (1977).

Legend of Figure $2 \mathrm{~B}$ at p. 71 should be changed to read:

B: Avoiding of uv light ( $370 \mathrm{~nm}$ ) as a result of step up (direct) photophobic responses (on-responses) at the border to the light. 